\title{
Determination of the minimum inhibitory concentration (MIC) and mutant prevention concentration (MPC) of selected antimicrobials in bovine and swine Pasteurella multocida, Escherichia coli, and Staphylococcus aureus isolates
}

\author{
Kateřina Nedbalcová, Kateřina Nechvátalová, Zdeňka Kučerová \\ Veterinary Research Institute, Brno, Czech Republic
}

Received July 10, 2014

Accepted November 26, 2014

\begin{abstract}
We compared the values of the minimum inhibitory concentration (MIC) and mutant prevention concentration (MPC) values of three antimicrobial agents for 72 bovine isolates of Pasteurella multocida, 80 swine isolates of P. multocida, 80 bovine isolates of Escherichia coli, 80 swine isolates of E. coli, and 80 isolates of Staphylococcus aureus from bovine mastitis. The ratio of $\mathrm{MIC}_{90} / \mathrm{MPC}_{90}$ which limited mutant selection window (MSW) was $\leq 0.12 / 4 \mathrm{mg} / 1$ for enrofloxacin, $0.5 / \geq 64 \mathrm{mg} / 1$ for florfenicol and $4 / \geq 128 \mathrm{mg} / 1$ for tulathromycin in bovine $P$. multocida isolates, $\leq 0.12 / 2 \mathrm{mg} / 1$ for enrofloxacin, $0.5 / \geq 64 \mathrm{mg} / 1$ for florfenicol and $4 / \geq 128 \mathrm{mg} / 1$ for tulathromycin in swine $P$. multocida isolates, $1 / 16 \mathrm{mg} / 1$ for enrofloxacin, $8 / \geq 64 \mathrm{mg} / 1$ for florfenicol and $8 / \geq 128 \mathrm{mg} / \mathrm{l}$ for tulathromycin in bovine $E$. coli isolates, $0.5 / 16 \mathrm{mg} / \mathrm{l}$ for enrofloxacin, $\geq 64 / \geq 64 \mathrm{mg} / \mathrm{l}$ for florfenicol and $8 / \geq 128 \mathrm{mg} / \mathrm{l}$ for tulathromycin in swine $E$. coli isolates, and $0.25 / 16 \mathrm{mg} / 1$ for enrofloxacin, $4 / \geq 64 \mathrm{mg} / 1$ for florfenicol and $4 / \geq 128 \mathrm{mg} / \mathrm{l}$ for tulathromycin in $S$. aureus isolates. These findings indicate that the dosage of antimicrobial agents to achieve serum concentration equal to or higher than MPC could reduce selection of resistant bacterial subpopulation.
\end{abstract}

Bacteria, antibiotics, resistance, $M I C, M P C, M S W$, cattle, pigs

Effective antimicrobial therapy is compromised by spreading bacterial resistance which has been detected worldwide at different levels also in breeding animals. The reduction of bacterial resistance over time is mostly associated with a reduction in the consumption of antimicrobials (Aarestrup et al. 2008). However, there are a number of bacteria with mutations that prevent the action of antimicrobial substances and cause the selection of a resistant mutant during clinical treatment in the bacterial population susceptible to antimicrobials (Canton and Morosini 2011).

The antimicrobial agents are used for therapy on the basis of the dosage strategy for antimicrobials according to their pharmacokinetic (PK) and pharmacodynamic (PD) properties and determination of minimum inhibitory concentration (MIC) for the pathogen. A new concept in the testing of antimicrobial resistance with the potential to reduce bacterial resistance is the dosing of antimicrobials based on their PK/PD properties and determination of the mutant prevention concentration (MPC) for the pathogen (Drlica 2001). The MPC is defined as the concentration of antimicrobial substances able to prevent the growth of resistant mutants with a density of inoculum $\geq 10^{9} \mathrm{CFU} / \mathrm{ml}$. The MPC is an indicator of susceptibility designed for a more accurate assessment of the potential of selection of a resistant mutant to antimicrobial substances than traditional methods for the determination of susceptibility (Zhao and Drlica 2008). It mostly better corresponds to the concentration of bacteria in the blood or in the target organ during acute infection of animals and people than MIC determination for inoculum with a density of $10^{5} \mathrm{CFU} / \mathrm{ml}$ (Croisier et al. 2004; Zhao and Drlica 2008). The concentration range between the MIC and the MPC is called the mutant selection window (MSW). When this window is wider, the risk of selection of resistant strains is also greater (Drlica 2001; Tam et al. 2005; Blondeau 2009).

Address for correspondence:

MVDr. Katerina Nedbalcova, Ph.D.

Veterinary Research Institute, Department of Immunology

Hudcova 70, 62100 Brno, Czech Republic
Phone: + 420533331217

Fax: +420541211229

E-mail: knedbalcova@centrum.cz;nedbalcova@vri.cz

http://actavet.vfu.cz/ 
The objective of this study was to compare the MIC and MPC values for enrofloxacin, florfenicol, and tulathromycin in E. coli, P. multocida, and $S$. aureus isolated from clinical cases of bovine or swine infections in the Czech Republic during the period from 2008 to 2012.

\section{Material and Methods}

Isolates

Isolates of enterotoxigenic $E$. coli were obtained from enteric infections of post-weaned calves (80 isolates) and piglets (80 isolates) with diarrhoea, $P$. multocida isolates from the lungs after necropsy of fattening cattle (72 isolates) and nursery or grower-finisher pigs ( 80 isolates) with respiratory disorders, and $80 \mathrm{~S}$. aureus isolates from milk of dairy cows suffering from mastitis over the period 2008-2012. All E. coli isolates were positive in testing for the presence of the gene coding the production of enterotoxin STa, according to Cheng et al. (2006). Pasteurella multocida isolates were identified by PCR (Townsend et al. 1998) a S. aureus isolates were identified biochemically using the commercial kit STAPHYTEST (Erba Lachema, Czech Republic). The samples originated from animals without previous antimicrobial therapy during the last 3 weeks and the isolates from the same herds were included in the monitoring of MICs and MPCs of antimicrobials, with a minimum interval of 6 months from the last collection.

\section{MIC, MPC and MSW determination}

Sussceptibility to antimicrobial substances was determined by assignment of MIC according to the standardised dilution micromethod of the Clinical and Laboratory Standards Institute (CLSI 2013a; 2013b) using commercial kits (Trek Diagnostics Systems Inc., England). The kit quality was controlled by the reference strains of $E$. coli ATCC 25922 and $S$. aureus ATCC 29213. The MIC values were defined as the lowest concentration of an antimicrobial agent inhibiting visible bacterial growth of culture with the density of $10^{5} \mathrm{CFU} / \mathrm{ml}$. Breakpoints of resistances were defined according to the CLSI and European Committee on Antimicrobial Susceptibility Testing (EUCAST 2013).

Determination of the MPC was made in duplicate by the dilution method according to CLSI (2013a; 2013b), using a modification of the previously published method (Blondeau 2001; Randall et al. 2004). Quality control of the experiment was performed with the reference strains of E. coli ATCC 25922 and S. aureus ATCC 29213. The MPC was determined as the lowest concentration of an antimicrobial agent inhibiting visible growth of bacterial culture with density of $10^{9} \mathrm{CFU} / \mathrm{ml}$.

The MSW was determined as the ranges of concentration between $\mathrm{MPC}_{90}$ and $\mathrm{MIC}_{90}$.

\section{Results}

Distribution of MICs for enrofloxacin, florfenicol, and tulathromycin in bovine and swine $P$. multocida isolates, bovine and swine $E$. coli isolates, and $S$. aureus isolates from dairy cows mastitis and calculation of the $\mathrm{MIC}_{50}$ and $\mathrm{MIC}_{90}$ values are in Table 1 . There are also comparative MPC distribution data for the isolates tested against the 3 antimicrobial agents and calculation of the $\mathrm{MPC}_{50}$ and $\mathrm{MPC}_{90}$ values. Table 2 shows the percentages of susceptible, intermediately susceptible, and resistant $P$. multocida, E. coli, and S. aureus isolates to enrofloxacin, florfenicol, and tulathromycin. The ranges between $\mathrm{MIC}_{90}$ and $\mathrm{MPC}_{90}$ that present the MSW for enrofloxacin, florfenicol, and tulathromycin are also shown.

As detected by the CLSI (2013a; 2013b) and EUCAST (2013) methods for MIC determination the majority of the tested bovine and swine $P$. multocida isolates were susceptible to the tested antimicrobial substances with $\mathrm{MIC}_{90}$ values below the breakpoint of resistance. On the other hand, bovine and swine $E$. coli isolates and $S$. aureus isolates from dairy cow mastitis were mostly resistant or intermediately susceptible to florfenicol. The $\mathrm{MIC}_{90}$ values for all three antimicrobials were the same in bovine and swine P. multocida isolates and $S$. aureus isolates, but differences were found between bovine and swine $E$. coli, except for tulathromycin. The $\mathrm{MIC}_{90}$ value for enrofloxacin was slightly higher in bovine $E$. coli isolates (bovine isolates $1 \mathrm{mg} / 1$ and swine isolates $0.5 \mathrm{mg} / \mathrm{l}$ ) and marked difference (bovine isolates $8 \mathrm{mg} / \mathrm{l}$ and swine isolates $\geq 64 \mathrm{mg} / \mathrm{l}$ ) was detected for florfenicol. The MPC values were above the susceptibility/resistance breakpoints of enrofloxacin, florfenicol, and tulathromycin in all cases. Even for florfenicol and tulathromycin, the $\mathrm{MPC}_{50}$ values 


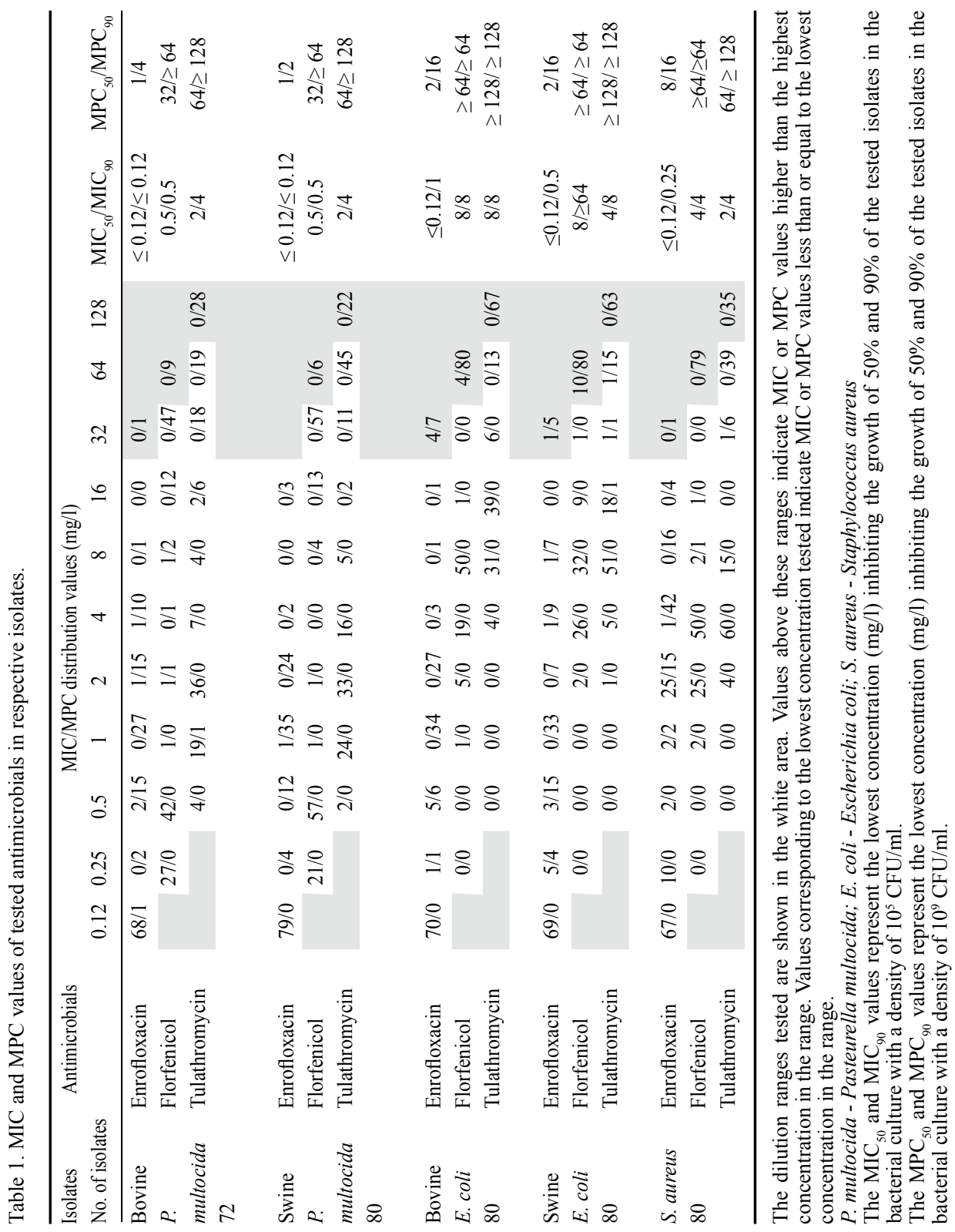

in all E. coli and S. aureus isolates and the $\mathrm{MPC}_{90}$ values in all tested isolates were above the range of concentrations.

We found quite wide ranges of concentrations between $\mathrm{MIC}_{90}$ and $\mathrm{MPC}_{90}(\mathrm{MSW}$ (Table 2). In most cases, they included the whole range of antimicrobials from the lowest tested concentrations under breakpoints of resistance to very high concentrations of antimicrobial agents, most often 


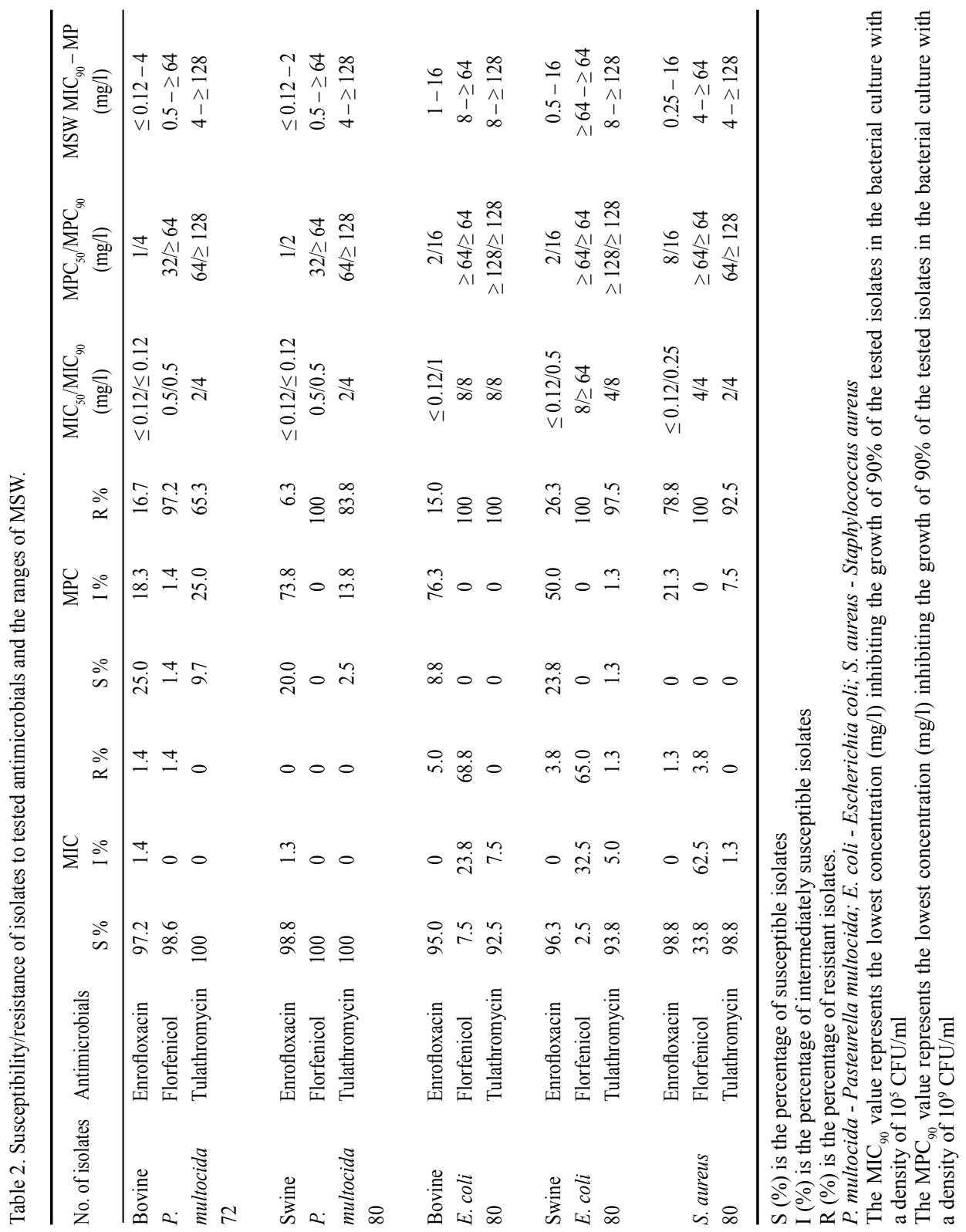

exceeding the highest tested concentration. The widest MSW were found in P. multocida isolates for florfenicol $(0.5-\geq 64 \mathrm{mg} / \mathrm{l}$ in bovine and swine isolates). It was possible to specify the width of MSW only for enrofloxacin where the values of $\mathrm{MPC}_{90}$ were within the tested ranges of concentration, but unfortunately, all $\mathrm{MPC}_{90}$ values were $\geq 64 \mathrm{mg} / \mathrm{l}$ for florfenicol and $\geq 128 \mathrm{mg} / \mathrm{l}$ for tulathromycin. Nevertheless, these not exactly defined values also represent the risk of the 
selection of resistance, because the MICs values of tested isolates (low limiting concentration of MSW) were low and the isolates were susceptible to the tested antimicrobials, except for florfenicol (all E. coli isolates) and enrofloxacin (swine E. coli isolates).

\section{Discussion}

To establish effective risk management measures, objective and valid data on the development, selection and spread of resistance in bacteria are essential. The data collected in veterinary monitoring studies should allow us to (1) recognize the emergence of new resistance phenotypes, (2) determine time-dependent trends in the development of resistance, (3) deduce epidemiological correlation regarding the present situation, (4) assess the development and spread of antimicrobial resistance in relation to antimicrobial usage, and thereby (5) to estimate the risk in veterinary medicine. With regard to the possible impact on human health, it is important to assess the risk of antimicrobial resistance caused by the use of antimicrobials in veterinary medicine (Wallmann 2006). The susceptibility or resistance of isolates are determined by using the standardized methods (CLSI 2013a; 2013b; EUCAST 2013), among them being the MIC determination. However, in recent studies, some authors have also recommended MPC determination and its comparison with MIC for prediction of selection and spread of antimicrobial resistances (Hesje et al. 2007; Lees et al. 2008; Blondeau 2009).

This manuscript provides information about testing the MIC and MPC in clinical isolates of some bovine and swine pathogens for selected veterinary antimicrobials. In all cases, the MPC values were much greater than the value of the MIC that was found in the majority of isolates. These results are in accordance with previous studies of the spread of resistance to antimicrobial agents due to selective pressure during clinical treatment (Hesje et al. 2007; Blondeau 2009). This is because the growth of susceptible bacterial populations is inhibited by antimicrobials. However, the therapeutic dose is not able to prevent further reproduction of mutant bacteria (Lees et al. 2008; Blondeau 2009). The MPC values of antimicrobials can be used clinically to reduce the development of resistance by different dosing strategies together with the assessment of other PK/PD indicators. Information on certain pharmaceuticals is available also in package leaflets (the value, the speed of achieving the maximum concentration of antimicrobial substances, and the dynamics of its decrease in serum and different tissues). In order to avoid the spread of a resistant mutant in a bacterial population, the concentration of antimicrobial substances in serum should reach the MPC value provided that it is safe for the treated animal. These values may vary between different species of animals (Blondeau 2009).

When we compare the information on $\mathrm{PK} / \mathrm{PD}$ properties of the tested antimicrobials (available on the drug manufacturer's website), the detected $\mathrm{MPC}_{90}$ values are higher than the highest concentration reached at the infection site after administration of the recommended dose of antimicrobials for treatment $\left(\mathrm{C}_{\max }\right)$. The $\mathrm{C}_{\max }$ of enrofloxacin is 0.49 $\mathrm{mg} / \mathrm{l}$ after administration of a dose of $2.5 \mathrm{mg} / \mathrm{kg}$ for cattle and $1.19 \mathrm{mg} / \mathrm{l}$ after the same dose for pigs. The $\mathrm{C}_{\max }$ of florfenicol is $3.37 \mathrm{mg} / \mathrm{l}$ after administration of a curative dose of $20 \mathrm{mg} /$ $\mathrm{kg}$ (cattle) and $5 \mathrm{mg} / \mathrm{l}$ after admninistration of a dose of $10 \mathrm{mg} / \mathrm{l}$ (pigs). The detected $\mathrm{MIC}_{90}$ values for enrofloxacin and florfenicol were lower or similar to their $\mathrm{C}_{\max }$ values, except for the majority of E. coli isolates. The $\mathrm{MPC}_{90}$ values (the upper limit of MSW) exceeded it in all cases. For tulathromycin, relatively low serum drug concentrations is characteristic (Nowakowski et al. 2004). The $\mathrm{C}_{\max }$ after administration of the recommended curative dose of $2.25 \mathrm{mg} / \mathrm{kg}$ was $0.5 \mathrm{mg} / \mathrm{l}$ for cattle and $0.6 \mathrm{mg} / \mathrm{l}$ for pigs and are below the $\mathrm{MIC}_{90}$ value detected in this study, similarly to other reports (Godinho et al. 2005; Blondeau et al. 2012). 
Based on the MIC and MPC of enrofloxacin, florfenicol, and tulathromycin, we identified the MSW of P. multocida and E. coli isolates from respiratory and enteric diseases of cattle and pigs and $S$. aureus isolates of dairy cattle mastitis to predict the likelihood of resistance selection. In this report, majority of isolates were susceptible or intermediately susceptible to all tested antimicrobials according to MIC determination, whereas the MPC values were usually high above breakpoints of susceptibility/resistance and the MSWs were detected as wide. Due to the fact that all tested bacteria may cause diseases of various animal species, including humans, these findings represent a major risk for the spread of resistant human and animal bacterial populations.

\section{Acknowledgements}

The authors wish to thank Mr. Paul Veater (Bristol, United Kingdom) and Mrs. Ing. Ludmila Faldikova, CSc. for proofreading the translated manuscript.

This work was supported by the Ministry of Agriculture of the Czech Republic (grant number QJ1210119) and the Ministry of Education, Youth and Sports of the Czech Republic (grant number LO1218). The results of the project LO1218 were obtained with a financial support from the MEYS of the CR under the NPU I program.

\section{References}

Aarestrup F, Duran CO, Burch GS 2008 Antimicrobial resistance in swine production. Anim Health Res Rev 9: $135-148$

Blondeau JM 2001: Clinical utility of the new fluoroquinolones for treating respiratory and urinary tract infections. Expert Opin Invest Drugs 10: 213-237

Blondeau JM 2009: New concepts in antimicrobial susceptibility testing: the mutant prevention concentration and mutant selection window approach. Vet Dermatol 20: 383-396

Blondeau JM, Borsos S, Blondeau LD, Blondeau BJJ, Hesje CE 2012: Comparative minimum inhibitory concentrations of enrofloxacin, ceftiofur, florfenicol, tilmikosin and tulathromycin against bovine clinical isolates of Mannheimia haemolytica. Vet Microbiol 160: 85-90

Canton R, Morosini MI 2011: Emergence and spread of antibiotic resistance following exposure to antibiotics. FEMS Microbiol Rev 35: 977-991

Cheng D, Sun H, Xu J, Gao S 2006: PCR detection of virulence factor genes in Escherichia coli isolates from weaned piglets with oedema disease and/or diarrhoea in China. Vet Microbiol 115: 320-328

CLSI 2013a: Performance Standards for Antimicrobial Disk and Dilution Susceptibility Tests for Bacteria Isolated from Animals; Approved Standard - Fourth Edition. CLSI document Vet 01-A4. Clinical and Laboratory Standards Institute, Wayne, PA 2013

CLSI 2013b: Performance Standards for Antimicrobial Disk and Dilution Susceptibility Tests for Bacteria Isolated from Animals; Second Informational Supplement. CLSI document Vet 01-S2. Clinical and Laboratory Standards Institute, Wayne, PA 2013

Croisier D, Etienne M, Bergoin E, Charles PE, Lequeu C, Piroth L, Portier H, Chavanet P 2004: Mutant selection window in levofloxacin and moxifloxacin treatments of experimental pneumococcal pneumonia in a rabbit model of human therapy. Antimicrob Agents Chemother 48: 1699-1707

Drlica K 2001: A strategy for fighting antibiotic resistance. ASM News 67: 27-33

EUCAST 2013: Antimicrobial wild type distributions of microorganisms. Version 5.13. [Database online]. European Committee on Antimicrobial Susceptibility Testing. Available at: www.eucast.org. Accessed September 26, 2013

Godinho KS, Keane SG, Nanijani IA, Benchaoui HA, Sunderland SJ, Jones MA, Weatherley AJ, Gootz TD, Rowan TG 2005: Minimum inhibitory concentrations of tulathromycin against respiratory bacterial pathogens isolated from clinical cases in European cattle and swine and variability arising from changes in in vitro methodology. Vet Ther 6: 113-121

Hesje C, Tillotson GS, Blondeau JM 2007: MICs, MPCs and PK/PDs: a match (sometimes) made in hosts. Expert Rev Resp Med 1: 7-16

Lees P, Svendsen O, Wiuff C 2008: Chapter 6. Strategies to minimise the impact of antimicrobial treatment on the selection of resistant bacteria. In: Guardabassi L (Ed): Guide to Antimicrobial Use in Animals 2008. Blackwell Publishing, Oxford, pp 77-101

Nowakowski MA, Inskeep PB, Risk JE, Skogerboe TL, Benchaoui HA, Meinert TR, Sherington J, Sunderland SJ 2004: Pharmacokinetics and lung tissue cocentrations of tulathromycin, a new triamilide antibiotic, in cattle. Vet Ther 5: 60-74

Randall L, Cooles S, Piddock L, Woodward M 2004: Mutant prevention concentrations of ciprofloxacin and enrofloxacin for Salmonella enterica. J Antimicrob Chemother 54: 688-691

Tam VH, Louie A, Deziel MR, Liu W, Leary R, Drusano GL 2005: Bacterial-population responses to 
drug-selective pressure: examination of garenoxacin's effect on Pseudomonas aeruginosa. J Infect Dis 192: 420-428

Townsend KM, Frost AJ, Lee CW, Papadimitriou JM, Dawkins HJS 1998: Development of PCR assays for species and type specific identification of Pasteurella multocida isolates. J Clin Microbiol 36: 1096-1100

Wallmann J 2006: Monitoring of antimicrobial resistance in pathogenic bacteria from livestock animals. Int J Med Microbiol 296: 81-86

Zhao X, Drlica K 2008: A unified anti-mutant dosing strategy. J Antimicrob Chemother 62: 434-436 